\title{
De la dissimulation du sens à la protection des données : petite histoire de la cryptologie
}

\section{Valérie Caniart}

\section{(2) OpenEdition}

1 Journals

Édition électronique

URL : http://journals.openedition.org/rbnu/1482

DOI : 10.4000/rbnu.1482

ISSN : 2679-6104

\section{Éditeur}

Bibliothèque nationale et universitaire de Strasbourg

\section{Édition imprimée}

Date de publication : 1 mai 2016

Pagination : 24-35

ISBN : 9782859230623

ISSN : 2109-2761

\section{Référence électronique}

Valérie Caniart, « De la dissimulation du sens à la protection des données : petite histoire de la cryptologie », La Revue de la BNU [En ligne], 13 | 2016, mis en ligne le 01 mars 2020, consulté le 12 décembre 2020. URL : http://journals.openedition.org/rbnu/1482 ; DOI : https://doi.org/10.4000/rbnu. 1482

\section{(c) (†)(2)}

La Revue de la BNU est mise à disposition selon les termes de la Licence Creative Commons Attribution - Pas d'Utilisation Commerciale - Partage dans les Mêmes Conditions 4.0 International. 


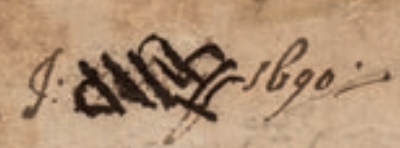

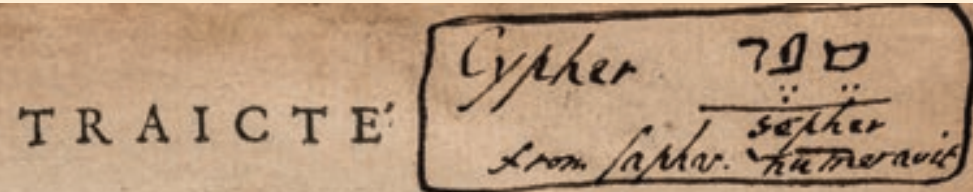

$761 \mathrm{DES}$. C H F F E S,

\section{O V SECRETES}

- MANIERES

D'ESCRIRE:

P A R

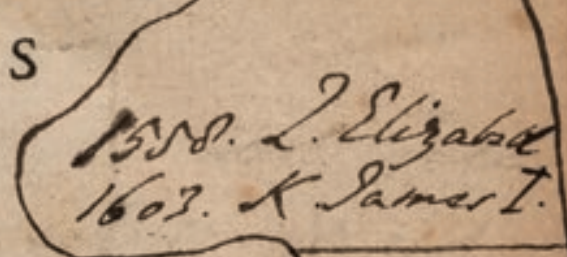

- BLAISE DE VIGENERE) Truac

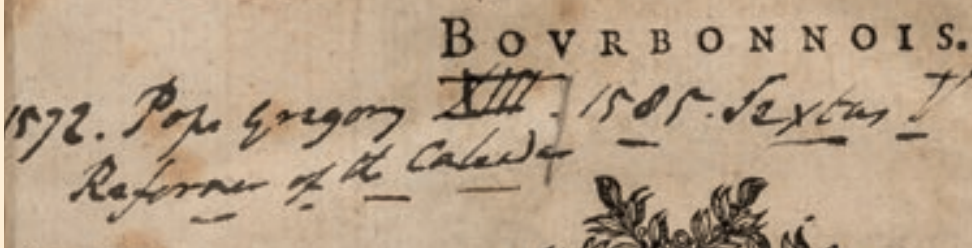
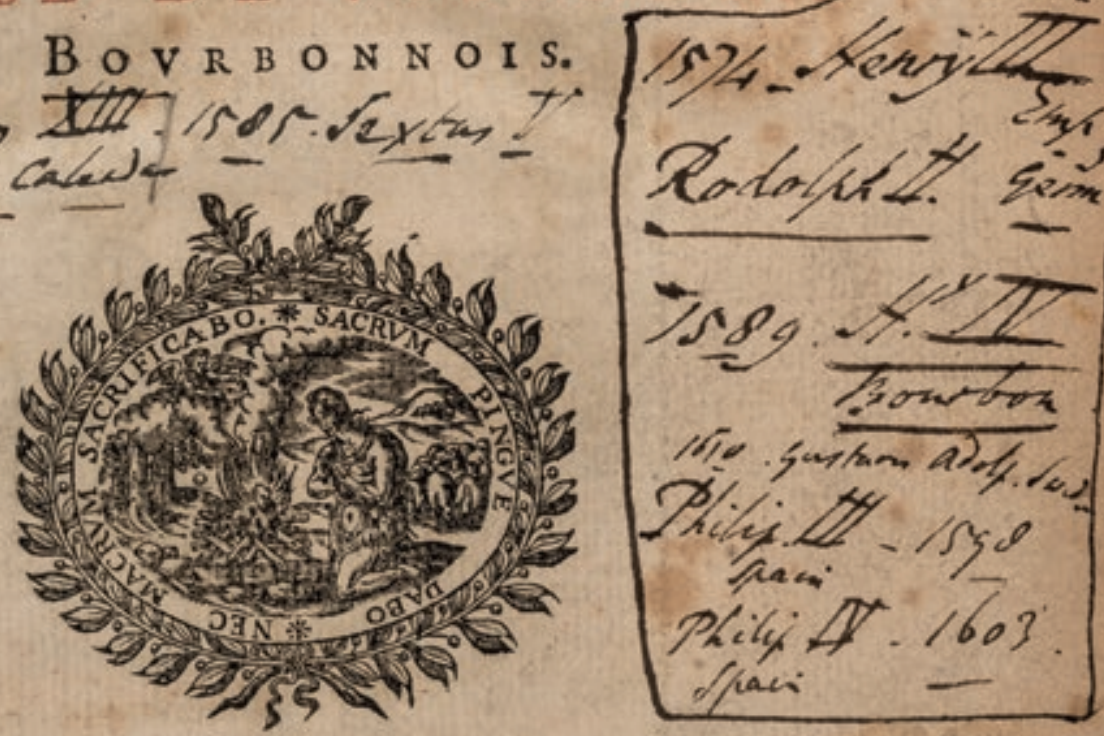

A P A R I $S$,

Chez A BEL L'A NGELTER, au premier pillier de la grand' Salle du Palais.

$$
\text { TH. D. } L X \times \times V Y \text {. }
$$

AVEC PRIYILEGE DV KOY.

$6:-$

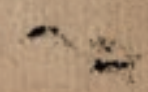

Page de titre du Traicté des chiffres, ou secrètes manières d'escrire de Blaise de Vigenère (Paris, L'Angelier, 1586 ; coll. BNU) 


\section{DE LA DISSIMULATION DU SENS À LA PROTEGTION DES DONNÉES : petite histoire de la cryptologie}

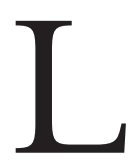

a cryptographie, ou l'art de dissimuler le sens d'un texte à l'attention d'une tierce personne, est une pratique presque aussi ancienne que l'écriture elle-même. Il semblerait que le texte au sens dissimulé le plus ancien qui ait été retrouvé soit de nature économique : il s'agit d'une tablette mésopotamienne datant d'environ 1500 av. J.-C. et recelant la formule d'un vernis à poteries. Ainsi, dès la plus haute Antiquité, l'homme a tenté de dissimuler des informations de toute nature : politique, militaire, économique. C'est toutefois dans les sphères politiques que ce stratagème a été le plus utilisé, à partir du $16^{\text {e }}$ siècle, date à laquelle les fondements de cette science ont été posés.

Aujourd'hui, cet usage est extrêmement répandu. La cryptographie n'est plus réservée aux seules sphères du pouvoir et de la diplomatie, mais elle est utilisée quotidiennement par tous ceux qui ont des informations à protéger, les entreprises comme les particuliers. Ainsi, à l'ère d'Internet et des objets connectés, les transactions des utilisateurs de la Toile sont protégées par des procédés cryptographiques avancés. Les techniques de camouflages se sont tellement complexifiées qu'elles sont devenues un domaine des mathématiques à part entière. Enfin, depuis le $20^{e}$ siècle, on ne parle plus de cryptographie mais de cryptologie.

Cette science nouvelle à l'histoire très ancienne est encore méconnue. L'historiographie est très récente puisqu'elle commence avec l'ouvrage, encore inégalé, de l'historien américain David Kahn, Code Breakers, the Story of Secret Writings, paru en 1967. Le sujet est relativement difficile à traiter et il suffit de parcourir les nombreux ouvrages pour s'en apercevoir. Se limiter à un aperçu historique fait prendre le risque d'énumérer de nombreuses anecdotes, la plupart relativement bien connues, mettant en scène le rôle joué par des messages décryptés qui ont changé le cours de l'Histoire, ou du moins l'issue d'une bataille, ou encore favorisé un retournement de situation, dans le but avoué de montrer l'importance de cette pratique de l'ombre. L'autre travers serait de tomber dans un discours purement technique, décrivant les différents procédés de chiffrement. Il en résulterait un discours austère et indigeste, décourageant très vite le non-initié.

À travers les trois grandes périodes reconnues par les spécialistes, que Jacques Stern a qualifiées de période artisanale, période mécanique et ère moderne ${ }^{1}$, et tout en reprenant la mise en garde de David Kahn, de " [...] veiller à ne pas attribuer à la seule cryptographie tout le mérite d'une victoire ou d'un succès diplomatique ", l'objectif de cet article est de montrer que l'évolution des techniques de chiffrement accompagne en fait celle de toute société qui s'épanouit sur les échanges et le partage de l'information.

\section{L'ère artisanale ou la cryptographie manuelle}

La première période, très longue, s'étend sur plusieurs siècles, de l'Antiquité à la veille de la Première Guerre mondiale. Il s'agit en fait d'une longue maturation et de mises au point de procédés et principes qui se sont développés de façon très sporadique. On peut parler de cryptographie manuelle, parce que la seule ressource est l'ingéniosité et la puissance de calcul des hommes chargés de développer et d'appliquer les méthodes de 
chiffrement. Cette période peut elle-même être découpée en trois temps : l'Antiquité, un premier sursaut aux $16^{\mathrm{e}}-17^{\mathrm{e}}$ siècles, puis l'essor définitif dans la seconde partie du $19^{e}$ siècle.

De l'Antiquité, trois procédés sont parvenus jusqu'à nous à travers quelques écrits attestant de leur existence et de leur emploi : la scytale des Lacédémoniens, le carré de Polybe et l'alphabet de Jules César.

La première est décrite par l'historien Thucydide comme ayant été inventée à Sparte au $4^{\mathrm{e}}$ siècle avant notre ère. Il s'agit d'un bâton cylindrique en bois d'un diamètre déterminé, dont la surface est taillée de façon à former plusieurs facettes. Une longue bandelette de cuir, tissu ou papyrus est ensuite enroulée autour. Le message est inscrit en suivant les facettes du cylindre. La bandelette déroulée ne laisse donc plus apparaître qu'une suite de lettres sans signification. L'historien Polybe, quant à lui, invente au $2^{e}$ siècle av. J.-C. un procédé de chiffrement connu sous le nom de carré de Polybe ou carré de 25. Il s'agit d'une grille de 25 cases (mais on peut aller jusqu'à 36 pour y faire figurer des chiffres), dont on numérote la première ligne et la première colonne. Le reste est rempli des lettres de l'alphabet. Le principe est de remplacer chaque lettre par ses coordonnées dans le tableau. Enfin, les écrits de Jules César laissent entendre que celui-ci utilisait comme moyen de chiffrement un alphabet décalé, appelé depuis alphabet de César. Mais en dehors de ces traces épistémologiques attestant de pratiques de chiffrement de textes, il ne reste aucun témoignage de théorisation sur le chiffre.

Après la chute de l'empire romain d'Occident et pendant tout le haut Moyen Âge, les historiens s'accordent pour dire que ces procédés tombent dans l'oubli. L'une des raisons est probablement le recul de l'écrit. À l'image de Charlemagne, dont la légende dit qu'il ne savait pas lire, la plupart des seigneurs formant l'élite guerrière sont illettrés ; la lecture et l'écriture sont l'apanage des seuls ecclésiastiques. L'écriture constitue par elle-même un moyen de chiffrement, tandis que l'Église condamne tout usage de chiffre. Les connaissances héritées de l'Antiquité sont perpétuées dans le monde arabe. Le plus ancien texte connu décrivant une méthode de décryptement est rédigé au $9^{\mathrm{e}}$ siècle par le philosophe al-Kindi ${ }^{2}$, connu également sous le nom latinisé d'Achindius.

C'est sans doute par ce biais qu'est redécouverte cette technique en Occident au $15^{\mathrm{e}}$ siècle, d'abord en Italie, dans les États du pape, la République de Venise et dans les puissantes cités de Milan ou Mantoue, puis en France et dans une moindre mesure en Allemagne et en Angleterre. Il est intéressant de noter l'apparition au $15^{\mathrm{e}}$ siècle du terme " chiffre ", issu de l'arabe " sifr ". Il désigne l'ensemble des conventions qui permettent de transformer un texte " clair ", c'est-à-dire intelligible par tous, en texte " chiffré " donc illisible par qui ne possède pas la convention, et inversement. Par extension, le mot " chiffreur " fait son apparition dès le premier quart du $16^{\text {e }}$ siècle pour désigner celui qui transcrit un texte en clair ou en chiffré. Du $16^{\mathrm{e}}$ au $17^{\mathrm{e}}$ siècle, l'emploi de chiffres s'impose dans les relations diplomatiques et au plus haut niveau du commandement militaire. Ce développement aboutit à l'apparition du mot " cryptographie ", au début du $17^{e}$ siècle, mot qui signifie alors " science du chiffre" ou " art des écritures secrètes ", indiquant par là-même une certaine codification et normalisation des pratiques.

Ainsi, Alberti rédige ce qui peut être considéré comme le premier traité de cryptographie où est décrit un procédé de chiffrement par substitution, invente un cryptographe, le Cadran d'Alberti, et s'intéresse au déchiffrement en réalisant la première analyse linguistique sur la fréquence des lettres et des digrammes dans les langues latine et italienne. En Italie toujours, Jérôme Cardan (Gerolamo Cardano, 1501-1576) invente quant à lui la fameuse grille de Cardan, décrite ainsi par Edmond Lerville : "Cette grille comporte un certain nombre de fentes égal au quart des cases, disposées de telle manière que des rotations successives de $90^{\circ}$ découvrent successivement toutes les cases de la grille. Le message à transmettre est écrit successivement dans chaque portion de la grille. On obtient donc une transposition " 3 . Ces réflexions sont divulguées grâce aux livres et l'intérêt déborde largement des frontières.

En France, Blaise de Vigenère, après avoir étudié le chiffre à Rome lors d'un voyage en 1549, fait paraître un Traicté des chiffres, ou secrètes manières d'escrire en 1586, dans lequel il décrit un système de chiffrement par substitution à double clé qu'il appelle le système indéchiffrable. Il le resta en effet assez longtemps. Le déchiffrement est quant à lui étudié par François Viète (1540-1630), attaché au service d'Henri IV.

Enfin, il faut nommer Antoine Rossignol (1590-1673), féru de cryptographie et propriétaire d'une bibliothèque rassemblant tous les ouvrages de l'époque sur le sujet, appelé au service du Roi pour déchiffrer des dépêches huguenotes et anglaises. À la vue des résultats hautement satisfaisants (capitulation de la ville de Réalmont dans le Tarn en 1627 et de La Rochelle en 1628), Richelieu l'attacha définitivement à son service et lui demanda de créer 


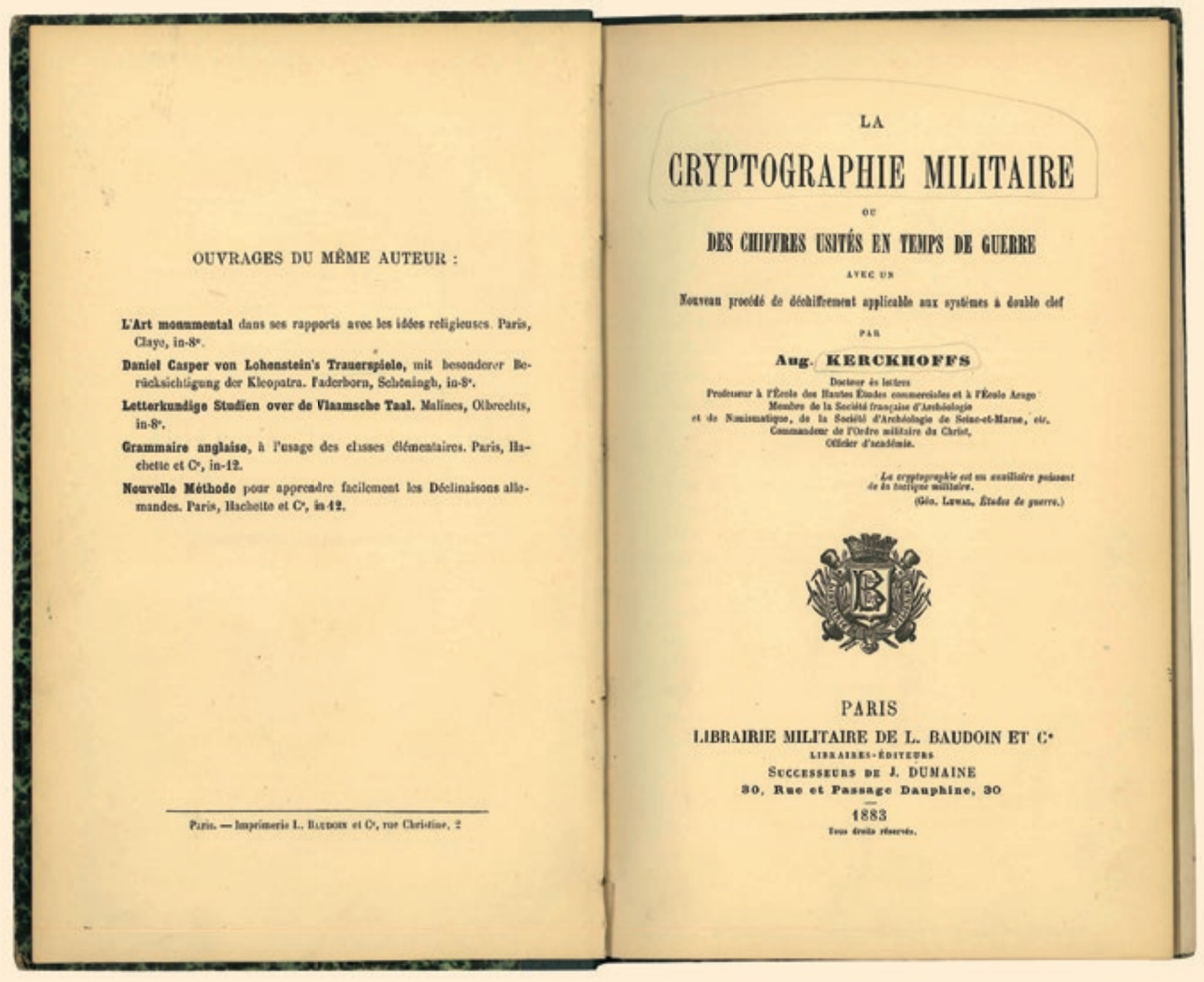

Première page du tiré-à-part de l'ouvrage d'Auguste Kerckhoffs. Celui-ci peut être considéré comme le premier ouvrage théorique du chiffre militaire en langue française (coll. Musée des transmissions). 
le premier service du chiffre en France ${ }^{4}$. Rossignol est l'auteur du Grand Chiffre de Louis XIV, resté indéchiffré jusqu'au début du $20^{\mathrm{e}}$ siècle. Sa légendaire efficacité dans le déchiffrement lui valut de donner son nom à ce petit instrument destiné à crocheter les serrures.

À travers leurs écrits, ces différents auteurs décrivent les principes de chiffrement sur lesquels repose toute méthode $^{5}$ : le principe de transposition, ou mélange des lettres du message, et celui de la substitution ou remplacement des lettres par d'autres signes. Ils s'intéressent tout autant au chiffrement qu'à la cryptanalyse, c'est-àdire au moyen de casser un chiffre.

Le contexte dans lequel se développe la cryptographie est tout aussi important. L'invention de l'imprimerie autour de 1453 révolutionne le monde occidental et accompagne le mouvement intellectuel de la Renaissance. Elle permet à une plus grande frange de la société d'accéder à l'écrit et de découvrir les textes des auteurs antiques, jusque-là réservés aux savants des monastères. Par conséquent, ce seul développement de l'écrit pourrait amener à penser qu'il devient nécessaire de dissimuler des informations susceptibles d'être interceptées par un plus grand nombre de personnes. Mais il est permis de penser que ce n'est pas une raison suffisante. Cette période de florissement intellectuel est également celle d'une expansion économique sans précédent, due à de multiples améliorations (techniques agricoles permettant une meilleure productivité, techniques de navigation favorisant l'expansion coloniale et commerciale, apparition de nouvelles structures financières, etc.) et dont l'aboutissement est l'émergence d'une nouvelle classe sociale, la bourgeoisie. Il existe donc un plus grand nombre de personnes détenant des informations ou des intérêts suffisamment importants pour vouloir les dissimuler à d'éventuels concurrents.

À partir de la seconde moitié du $17^{\mathrm{e}}$ siècle, le chiffre entre dans une période de déclin, suscitant peu d'études, ne révélant aucun auteur digne d'intérêt, tandis que la mise en œuvre des procédés de chiffrement tombe au plus bas, jusqu'à la première moitié du $19^{\mathrm{e}}$ siècle. S'ouvre alors la troisième et dernière période de cet âge artisanal, le dernier quart du $19^{\mathrm{e}}$ siècle.

Deux raisons principales favorisent le renouveau de la cryptographie, en particulier en France : l'invention et le développement du télégraphe et la réforme en profondeur de l'armée française après la défaite humiliante de 1870.

La pose du premier câble transatlantique en 1866 marque un tournant dans l'usage du télégraphe électrique. La circulation de messages de toutes natures, commerciales, financières, politiques, dont certains sont susceptibles de comporter des informations capitales, sur les lignes télégraphiques étrangères les mettent à la merci de toutes les indiscrétions. Cela fait réfléchir à la nécessité de protéger les informations. Mais plus encore, ce sont les tarifs d'expédition pratiqués par les compagnies télégraphiques exploitantes qui incitent à revoir les procédés de chiffrement. Une anecdote permet de prendre la mesure de cette contrainte économique.

En 1867, peu après l'établissement sur le trône du Mexique de Maximilien d'Autriche par des troupes françaises envoyées sur place, le secrétaire d'État américain William Seward expédie à Paris une dépêche demandant le retrait des troupes françaises. Le télégramme, chiffré au moyen du code diplomatique alors en usage dans les ambassades américaines, comporte 1100 groupes de 3, 4 ou 5 chiffres. Les télégrammes étant alors facturés à la lettre, le montant s'élève à $23000 \$^{6}$ ! Seward, refusant de payer une telle somme, entame des négociations pour obtenir des tarifs plus favorables à son administration. En France, le bureau du chiffre du ministère des Affaires étrangères est confronté au même problème et se donne pour objectif de baisser de $40 \%$ les frais d'expédition des télégrammes chiffrés ${ }^{7}$.

Du côté militaire, la guerre de 1870 a révélé deux défaillances dans le domaine des transmissions : l'inadaptation du service télégraphique et le manque de moyens de chiffrement qui dépendaient alors uniquement des Affaires étrangères. C'est donc avec des préoccupations d'emploi que les premiers théoriciens commencent à réfléchir au chiffre. Dans une étude critique sur l'armée et les réformes à y introduire, le général Lewal ${ }^{8}$ définit pour la première fois en 1881 le chiffre militaire et les besoins de 


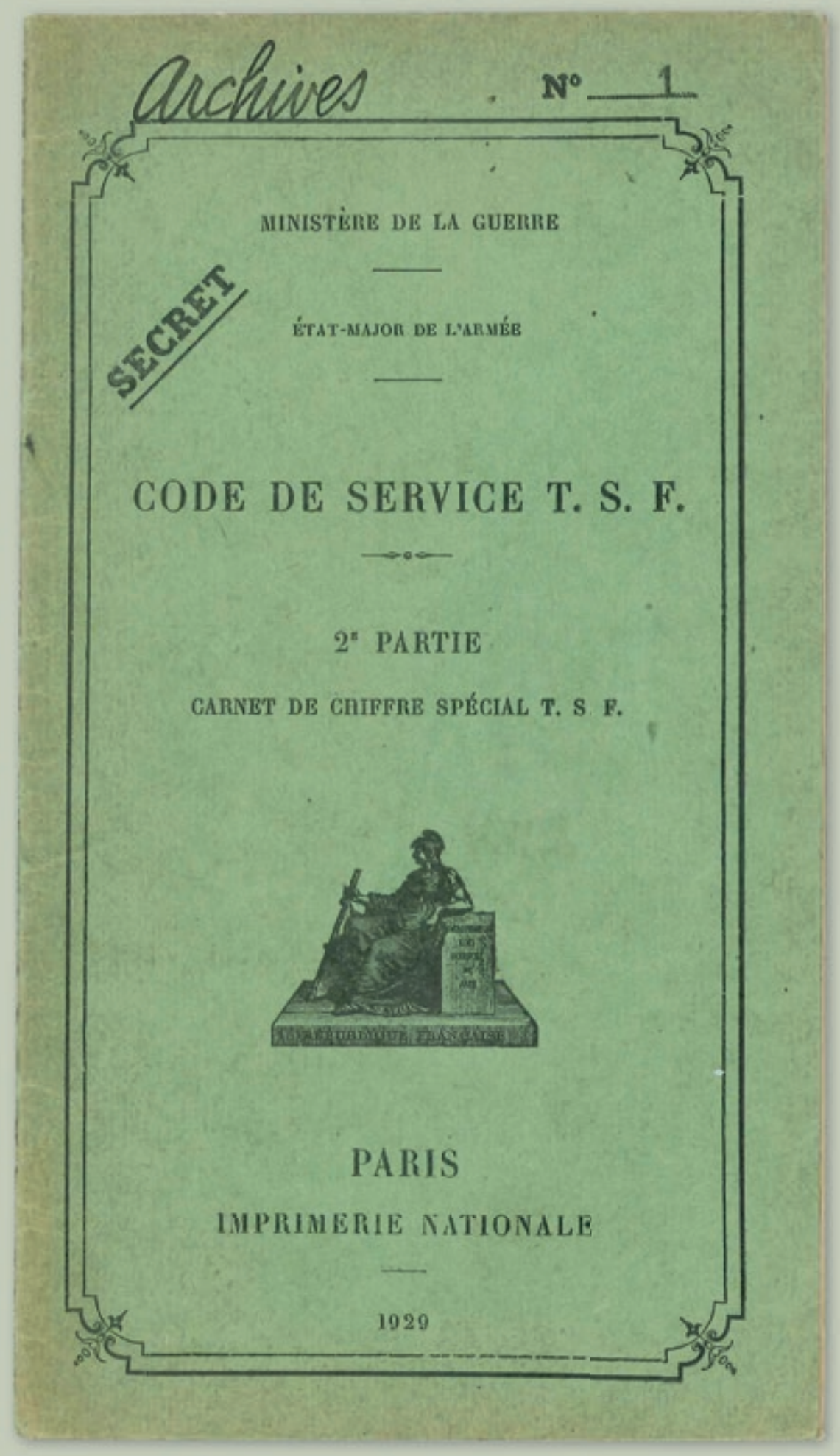

Page de couverture du code de service TSF en usage au ministère de la Guerre pour les transmissions d'ordre technique ou les radiotélégrammes de services (coll. Musée des transmissions). 
ebiffres maryuant ha pagnation $(01-02-63 . \ldots .80-40-41 \ldots \ldots$ $6 s-\infty=0$

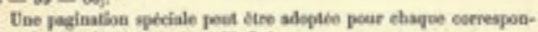
dant. On peut la taire narier 1 rinfui.

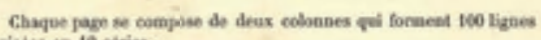
firisees en 10 stries.

la promiltre ligne de thagoe serie est commandet par be chillre 6.

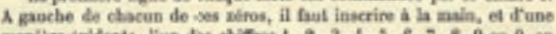

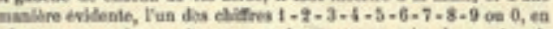
alopkant un oedro quelooeque, mais ea ayant soin de no jamais reprodtire be mitse ara tive sur la meine page.

la premilese Egre de chaque serie portara ainsi en the fren des notnderes suivatsts : $10-80-30 \ldots . . .80-90-60$.

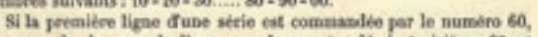
par esemple, la seosade ligne aura in numbiro 61 , 6 troisitme 62, ef ainsi de sulte.

Chague not oa exprowice sera repetsente par in groupe de cliffres forme de la stunios da ramere de la page et de eclui de la ligee ot se treure lo mot oe lexpressioe.

Lo nag occupt par ciseva des chiffos composant ee grovge sen

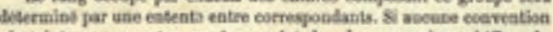
s'est intervesue edtre eux a co sujet, bes deux premiers ehifres du groupe repetsenteeost la page es les deux derniens la ligse.

La pagination pournil itre indlyute par des nombers coupoets de trois chiffres: Dans to eas, elaque mot serait exprime par ut greupe de 5 signaux.

On trouve a leur lettre slphabetique et impeimes en caractiones italiques les olgmes de posctuation (point, vingule, ofparation de deux woses syliables, ete.

On y trowe masi fixdication du gonre et da aomber des nots

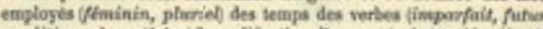

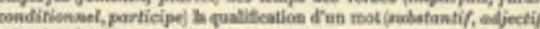

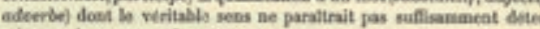
mine par le grouje qui repeisente ce mol.

Les groupes agurant ces felicatices dewnat etre plocta par lo

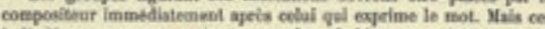
indications ne secost enp loyess qua dons de Elen rares circonstanoes :

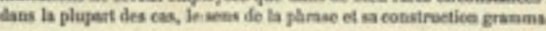

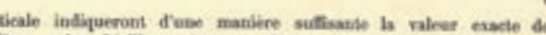

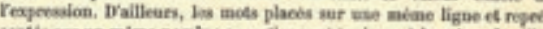

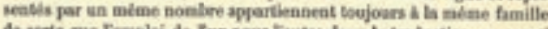

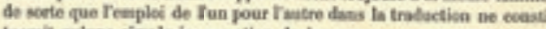

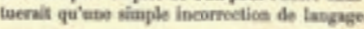

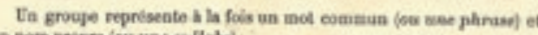
es notm propre (ou une splithbe).

Les moms perges et syllabes forment une eolotane supplenentsin droble do la colcano prircipalo.

Ea genteal, bens de la parase indigpera d'use maniere suft.

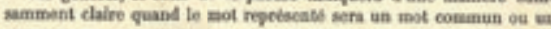
noan joopee.

Maik, si le moindre doate pounit exister dans la tratuction au sujet du mot emploge, te compositeur ne devnit pas besiler i falre prectife to nombre repetsentant bo now peipe ou la sylabe de fun des

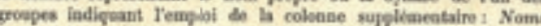

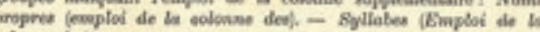
colossne des).

Au poiat de vue telegraplique, outse Tavadage que presente remploi fu souvesu codo pour le sccret de to comsependance, if a

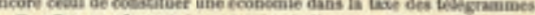

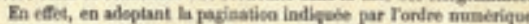
des feuillets of en numeratant les lignes 00 i 9 du haut en bas do bo

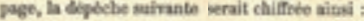

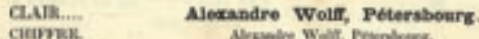

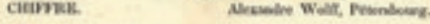

C.AIR.... Je vous prise de repodire telegrapalquement
chims.

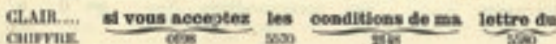

C.AIR... vingt-cing courabt. = Dopont.

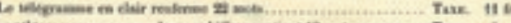

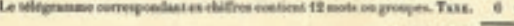

Extrait de l'instruction pour l'usage du vocabulaire administratif et commercial (in Nouveau code ou vocabulaire administratif et commercial pour la correspondance secrète, par Nilac, Paris, Librairie étrangère, 1891 ; coll. Musée des transmissions).

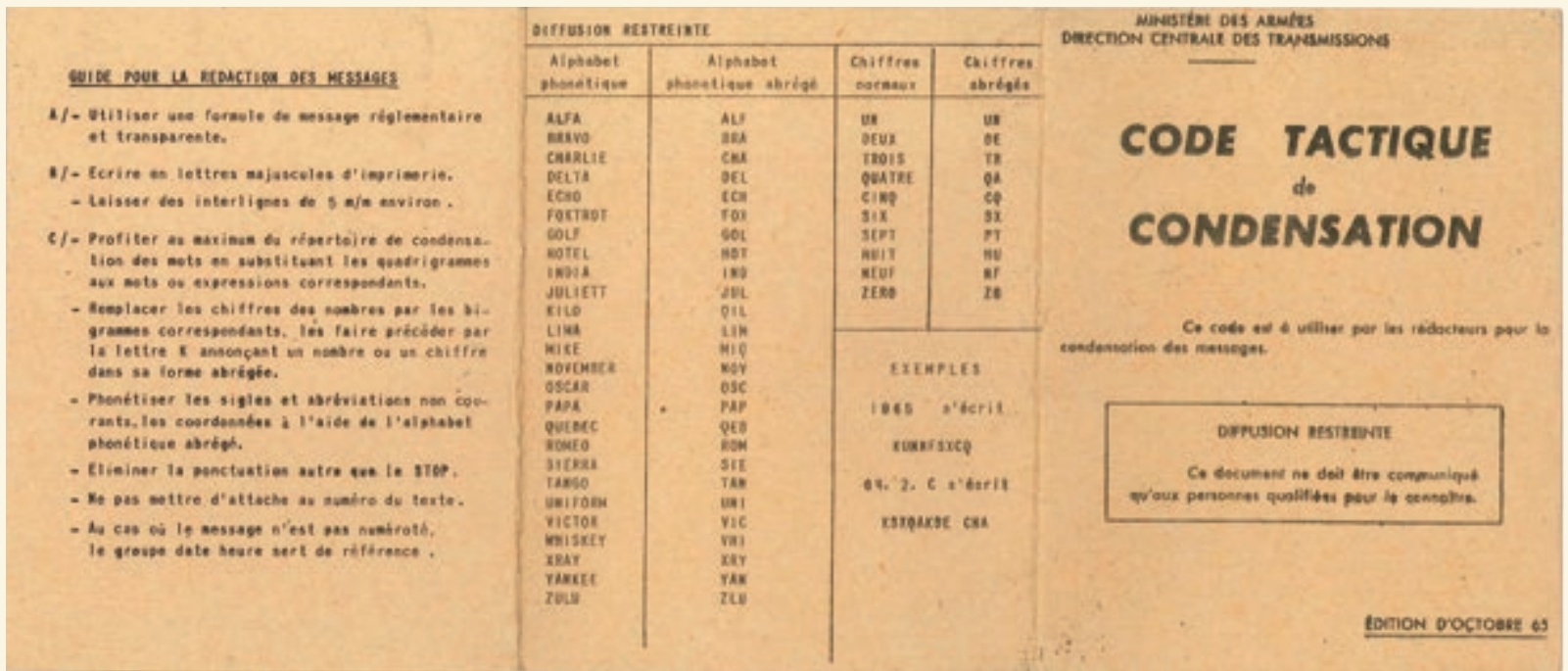

Code tactique de condensation, ministère des Armées, octobre 1965, dépliant cartonné imprimé sur 4 volets recto-verso, 10,5 x 13,5 cm. Il était destiné à être mis dans la poche pour les chiffreurs en manœuvres (coll. Musée des transmissions). 
l'armée en temps de guerre. Ses arguments sont repris et affinés en 1883 par Auguste Kerckhoffs ${ }^{9}$ dans un ouvrage consacré à la cryptographie militaire. Les études de Lewal et Kerckhoffs permettent d'asseoir des bases théoriques indispensables et des définitions claires. Une prise de conscience existe sur le fait que la mise en place et le respect de procédures garantissent autant la sécurité des transmissions que l'efficacité du système de chiffrement.

Les nombreuses études qui éclosent dans le dernier quart du $19^{\mathrm{e}}$ siècle permettent l'émergence de concepts clairs " qui permettent de distinguer le principe d'un mécanisme cryptographique des variations qui peuvent lui être données au jour le jour, par le choix de paramètres secrets d'un format concis [c'est la clef de chiffrement] $"{ }^{10}$. Les méthodes de cryptographie restent collées à la pratique et sont l'œuvre d'ingénieurs, de linguistes, de militaires, de policiers qui ont une approche pragmatique des procédés de chiffrement et s'inquiètent de leur adaptation à l'usage du télégraphe. Il s'agit donc d'une réponse à un souci d'adaptation technologique. Outre le ministère des Affaires étrangères, tous les grands services de l'État (ministère de la Guerre, de la Marine, de l'Intérieur, des Postes) se dotent de services de chiffres dont l'organisation, le fonctionnement et la déontologie commencent à être fixés.

\section{La cryptologie mécanique}

À la fin de la Première Guerre mondiale, il est devenu évident pour les responsables des états-majors que les méthodes " manuelles " de chiffrement et de déchiffrement ont atteint leurs limites. Le volume des communications par la télégraphie explose et laisse les services du chiffre des différents belligérants noyés sous les dépêches à déchiffrer. La complexification des procédés de chiffrement mobilise des équipes des cryptanalystes pendant des jours, parfois des semaines, avant de réussir à casser un chiffre. L'une des préoccupations devient alors la rapidité du chiffrement en mécanisant cette fonction.

Avant même la fin du conflit, des ingénieurs cherchent à mécaniser l'action de chiffrement et déposent des brevets. Ainsi, en 1917, l'américain Edward Hugues Hebern établit les plans d'une machine à roues. Dans le même temps, le Hollandais H. A. Koch dépose en octobre 1918 un brevet pour une machine similaire, suivi par le Suédois Arvid Damm en 1919. Deux de ces brevets seront repris par des industriels quelques années plus tard, et les machines qui en sont issues s'affronteront en équipant chacune l'un des camps opposés. La machine de Koch, produite par une entreprise allemande, deviendra Enigma ${ }^{11}$, tandis que le modèle de Damm est racheté par un Suédois, Boris Hagelin, qui fournit les armées françaises et américaines avec ses modèles.

De l'extérieur, ces machines sont assez semblables les unes aux autres. Le plus souvent de l'apparence et de la taille d'une machine à écrire, pesant un peu plus d'une dizaine de kilos, elles possèdent des caractéristiques communes : un clavier servant à taper le texte en clair ou en chiffré ; un système de roues mobiles et interchangeables fixées sur un axe, chacune dotée d'un alphabet dans le désordre. Il n'y a pas de moteur : c'est l'enfoncement des touches du clavier qui entraîne la rotation des roues à alphabet, d'où leur catégorisation en machines mécaniques. Il n'existe pas de système d'impression, tout au moins dans les premiers temps (cette possibilité n'apparaît qu'à la demande du bénéficiaire). Enfin, elles ne sont reliées à aucun système de transmissions.

L'efficacité de ces machines provient des innombrables possibilités de combinaisons de chiffrement offertes par le nombre de roues. C'est d'ailleurs pour cette raison qu'Enigma avait la réputation d'être inviolable : ses différentes modalités de câblage permettaient un nombre de possibilités équivalent à 5 suivi de douze zéros. Il est d'ailleurs intéressant de noter que le développement de ces machines est parallèle à celui des calculateurs. Au cours de la Seconde Guerre mondiale, la machine Colossus mise au point par Alan Turing à Bletchley Park, à la demande des services de renseignements britanniques, n'est autre qu'un calculateur super-puissant destiné à tester les innombrables possibilités de chiffres recueillis par les services d'écoutes de l'armée britannique. Jacques Stern souligne là le lien très fort existant entre la naissance de l'informatique et la cryptologie : " L'apparition des machines à chiffrer permet de se dégager des contraintes physiques et d'enchaîner des opérations multiples. Du coup, le besoin de théorie se fait de nouveau sentir et la parole est de nouveau rendue aux mathématiciens ${ }^{12}$.

Pendant la Première Guerre mondiale, on avait découvert le moyen d'écouter les conversations téléphoniques. Aussi faut-il noter l'effort de recherche et les premiers essais de cryptophonie, pour protéger les conversations entre Churchill et Roosevelt. Mais aucun des moyens développés ne fut satisfaisant avant la numérisation de la parole. Après la Seconde Guerre mondiale, le principal moyen de transmission de messages chiffrés reste la télégraphie. 


$$
1
$$




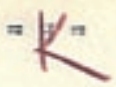

= GROTIPSS DE 5 TARVPES $=$ I JIII I9I8.

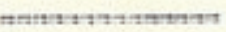

TEUPOLAาEL.

$\{5$

I5 $\mathrm{II} 20=\mathrm{CM}$ IOP $\mathrm{V}$ GTX $=I 525 \mathrm{cHI}$ I72 $=$ PFXDV AGPX GADDP XXAVG DXDVG DVADP XCXaA VAFFP GVGCD FFEA DADAD FFXAP QVGCQ AXVAX DGVDX YVAGA ZMGEA AXAAG
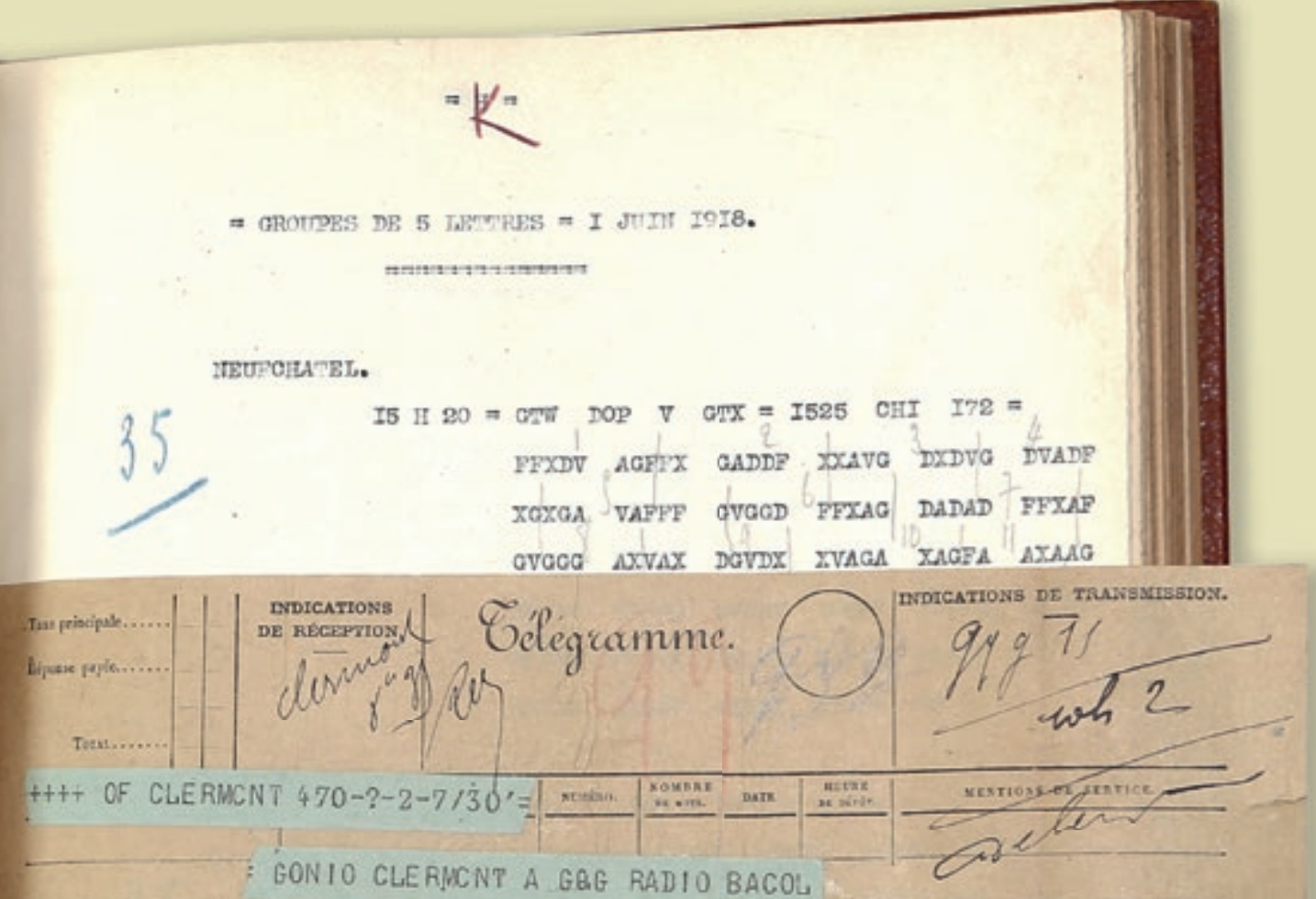

-2. H 22.- DDI V DSK -1945-CHI 58- ADXXV GGXFA FXFVX AXVXV

FFXAV GAXGX AGFGX XVVFV VAAXG GGAAX XDXXY AGA ++

$\frac{1}{3}$

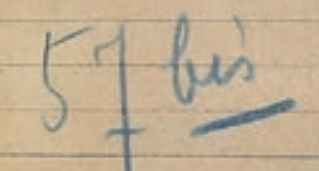

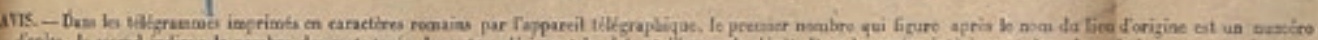

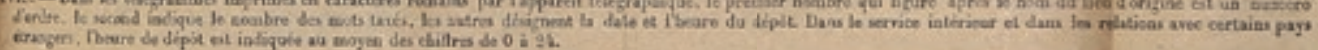

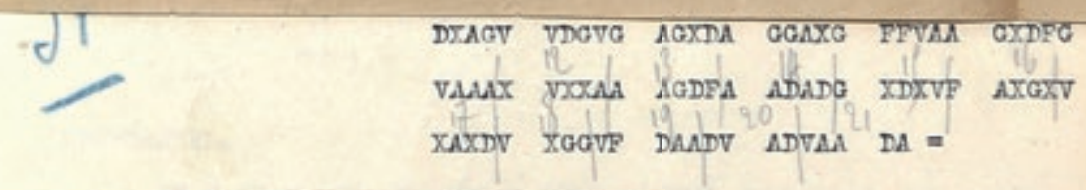

SOUILLY.

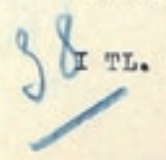

I5 II $33=$ DPO $\nabla$ DZO IKI3 2 TLE $=$

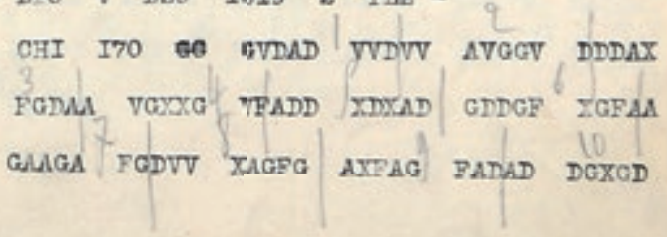


$\mathrm{Au}$ lendemain de cette guerre et pendant la guerre froide, le chiffre demeure un outil réservé à l'usage exclusif des États. De chaque côté du rideau de fer, les pays avancés en ce domaine engagent des moyens considérables pour le développement de puissantes mesures de cryptanalyse. Ces moyens sont d'ailleurs considérés comme du matériel de guerre. Les gouvernements de tous les pays ont gardé un silence presque total sur les moyens cryptologiques.

\section{La révolution technologique de 1976 : la cryptologie moderne}

À partir des années 1970, le développement de la cryptologie sort du domaine militaire et diplomatique pour tomber dans le domaine civil ou commercial. Cette évolution reste difficile à tracer du fait de la confidentialité des archives. L'une des principales raison en est le développement des échanges informatiques de nature financière et commerciale. Les milieux d'affaires en ressentent le besoin et exercent des pressions pour imposer la mise en place des moyens de protection de leur flux d'informations.

Le NBS (National Bureau of Standards) lance un appel à candidature pour un procédé susceptible d'être utilisé largement pour de telles transactions. IBM remporte la compétition en 1977 avec le " Data Encryption Standard ", qui est un algorithme reposant sur un procédé classique, la clé de chiffrement symétrique. Utilisé pour l'authentification de données, il devient l'algorithme le plus utilisé dans le monde : on le trouve dans les puces des cartes bancaires ou de la carte Vitale, les cartes SIM (Subscriber Identification Module) des téléphones cellulaires ou encore dans les décodeurs de télévision à péage comme celui de Canal +. En 1990, un nouvel algorithme de chiffrement symétrique est publié par Xuejia Lai et James Massey et exploité par la société Mediacrypt. C'est l'un des meilleurs systèmes à ce jour, qui n'a officiellement pas encore été décrypté.

La cryptologie est aujourd'hui un pilier d'un vaste ensemble, celui de la sécurité de systèmes d'informations. La nature des informations protégées a changé : il ne s'agit plus de protéger des informations confidentielles ou secrètes, mais de contribuer au bon fonctionnement d'installations ou d'infrastructures sensibles telles que des calculateurs utilisés dans les centrales d'énergie, les réseaux de communication ou de distribution, ou encore les circuits financiers, et que l'on dénomme également des infrastructures vitales ${ }^{13}$.

À travers cette évolution retracée à grands traits, on perçoit deux tendances. La première est une démocratisation et une banalisation progressives. D’abord réservée au cercle étroit des plus hautes autorités de l'État dans les seuls domaines de la diplomatie ou des affaires militaires, la nécessité de protéger les informations s'étend désormais à tous les domaines de l'activité humaine, depuis le chef de l'État jusqu'au simple consommateur dans ses transactions quotidiennes. On peut donc considérer que la protection de l'information est une condition incontournable, inhérente à la société dans laquelle nous vivons, où l'information est devenue une ressource comme une autre, parce qu'elle permet d'établir un ingrédient indispensable à tout échange : la confiance.

\section{Valérie Caniart}




\section{ORIENTATIONS BIBLIOGRAPHIQUES :}

- Arboit, Gérald, L'émergence d'une cryptographie militaire en France, note historique $\mathrm{n}^{\circ}$ 15, juillet 2008 (www.cf2r.org)

- Caniart, Valérie, "Écritures cachées, écritures codées : guerre et stratégie ", in Cacher Coder, 4000 ans d'écritures secrètes, Figeac, Musée Champollion-Les écritures du monde, 2015

- Ceillier, Rémi, La cryptographie, Paris, PUF, coll. "Que sais-je ? ", 1948

- Eyraud, Charles, Précis de cryptographie moderne, $2^{\mathrm{e}}$ édition, 1959

- Fletcher Pratt, Murray, Histoire de la cryptographie. Les écritures secrètes depuis l'Antiquité jusqu'à nos jours, Paris, Payot, 1940

- Kerckhoffs, Auguste, La cryptographie militaire, ou Des chiffres usités en temps de guerre, Paris, Librairie militaire L. Baudoin, 1883

- Lerville, Edmond, Les cahiers secrets de la cryptographie. Le chiffre dans l'histoire, des histoires du chiffre, Monaco, Éditions du Rocher, 1972

— Lewal, Jules-Louis, Études de guerre. Tactique du renseignement, 2 vol., Paris, Librairie militaire L. Baudoin, 1881. Réédition en 2013

— Ministère de l'Intérieur (service du chiffre), Manuel d'instruction, $\mathrm{n}^{\circ} 142$

- Ministère de la Guerre, Notions elémentaires de cryptographie, exemplaire $n^{\circ} 115$, Paris, Imprimerie nationale, 1920

- Ollier, Alexandre, La cryptographie militaire avant la guerre de 1914, Panazol, Lavauzelle, 2002

- Secrétariat général du gouvernement, Étude cryptographique, $\mathrm{n}^{\circ}$ 1/50, 1955

- Secrétariat général du gouvernement, Terminologie interministérielle de cryptographie à l'usage des spécialistes du chiffre, $\mathrm{n}^{\circ}$ 1499, Paris, 1956

— Stern, Jacques, La science du secret, Paris, Odile Jacob, 1998

- Valerio, Paul, De la cryptographie : essai sur les méthodes de déchiffrement, Paris, Librairie militaire L. Baudoin, 1893

- Wrixon, Fred B., Langages secrets. Codes, chiffres et autres cryptosystèmes, Cologne, Könemann, 2000

\section{Notes}

1 - In La science du secret, 1998 (voir bibliographie)

2 - Ce texte, retrouvé en 1987 dans les Archives ottomanes d'Istanbul, décrit l'analyse fréquentielle des lettres d'un texte chiffré et explique que le nombre et la répartition des caractères (lettres ou symboles) dans un texte offre des pistes pour le décoder. Voir aussi, sur al-Kindi, l'article d'Hervé Lehning p. 46.

3- Edmond Lerville, Les cahiers secrets, p. 31-32 (voir bibliographie)

4 - Voir à ce sujet l'article d'Hervé Lehning, p. 46.

5 - Charles Eyraud, Précis de cryptographie moderne, p. 9 (voir bibliographie)

6 - Murray Fletcher Pratt, Histoire de la cryptographie..., p. 1198-99 (voir bibliographie)

7- Gérald Arboit, L'émergence d'une cryptographie militaire en France (voir bibliographie)

8 - Jules Lewal, Études de guerre. Tactique du renseignement... (voir bibliographie)

9 - Auguste Kerckhoffs, La cryptographie militaire... (voir bibliographie)

10- Jacques Stern, La science du secret (voir bibliographie)

11-Sur la machine Enigma, voir l'article de Christian Westerhoff et Thomas Weis p. 62 .

12- Jacques Stern, op. cit., p. 11

13- Jacques Aubert (sous la dir. de), Cryptologie : la science du secret, Cesson-Sévigné, AAMTAT, 2012, p. 2 